Published in final edited form as:

Drug Discov Today Dis Mech. 2013 June 1; 10(1-2): . doi:10.1016/j.ddmec.2013.03.006.

\title{
Obesity and metabolic inflammation
}

\author{
Haiyan Xu \\ Hallett Center for Diabetes and Endocrinology, Division of Endocrinology, Department of \\ Medicine, Rhode Island Hospital, Warren Alpert Medical School of Brown University, Providence, \\ RI 02903
}

\begin{abstract}
Obesity epidemics affect $35.7 \%$ of adults and approximately $17 \%$ of children in the United States. Obesity has been associated with several health disorders, such as type 2 diabetes, cardiovascular diseases, fatty liver disease, and certain forms of cancer. Medical costs associated with obesity were estimated at $\$ 147$ billion in 2008. Chronic tissue inflammation, particularly in adipose tissue, has been considered as a key underlying mechanism for the development of obesity-related metabolic syndrome. In this review, we discuss the recent progress in the field of metabolic inflammation and the potential implication of anti-inflammation approaches as therapeutic interventions for treating obesity-related metabolic disorders.
\end{abstract}

\section{Keywords \\ WAT; obesity; inflammation}

The incidence of obesity has increased dramatically in the United States during the past decade and has become an epidemic disease. Massive expansion of adipose tissue, the major site for storage of excessive energy in the form of triglycerides, is the hallmark of obesity. Obesity caused by genetic disorders only accounts for a very small portion. The main drive is excessive energy intake and lacking of physical activity. Extensive studies indicate that obesity might be an inflammatory disease originated in adipose tissue.

\section{A low grade chronic inflammation in obesity}

Human population studies have revealed a correlation between obesity and inflammation several decades ago yet the source of elevated circulating inflammatory markers remained unknown till identification of increased TNFa expression in obese adipose tissue about twenty years ago [1]. Other proinflammatory cytokines, including IL-1 $\beta$, and MCP-1, were subsequently found to increase in obese adipose tissue. The discovery of proinflammatory macrophage infiltration into obese adipose tissue reveals an important source for circulating inflammatory markers [2]. Adipose tissue expansion during obesity development occurs

\footnotetext{
(C) 2013 Elsevier Ltd. All rights reserved.

Corresponding to be addressed to: Haiyan Xu MD, PhD, Hallett Center for Diabetes and Endocrinology, Division of Endocrinology, Department of Medicine, Rhode Island Hospital, Warren Alpert Medical School of Brown University, 55 Claverick St., Rm 318, Providence, RI 02903, Tel. 401-444-0347, Fax. 401-444-3784, hxu@lifespan.org.

Conflict of interest

The author declares no conflict of interest.

Publisher's Disclaimer: This is a PDF file of an unedited manuscript that has been accepted for publication. As a service to our customers we are providing this early version of the manuscript. The manuscript will undergo copyediting, typesetting, and review of the resulting proof before it is published in its final citable form. Please note that during the production process errors may be discovered which could affect the content, and all legal disclaimers that apply to the journal pertain.
} 
mainly through enlarging the size of existing adipocytes. The pathological growth of adipose tissue in response to over-nutrition is associated with insufficient vascularization which results in poor oxygenation. Hypoxia has been considered as one potential factor for inducing adipose inflammation since increased expression of proinflammatory factors such as TNFa, IL-1 $\beta$ and MCP-1 have been observed in cultured and primary adipocytes. Dysregulation of lipolysis occurs in obese adipose tissue and elevated circulating levels of free fatty acids (FFAs) contribute to systemic insulin resistance (Figure 1). Proinflammatoty cytokines activate two major inflammatory kinases: Ikappa B kinase $\beta$ (IKK $\beta$ ) and c-Jun Nterminal kinase (JNK). Both kinases have been shown to phosphorylate Ser307 of insulin receptor substrate 1 (IRS-1), a mechanism for attenuating insulin-stimulated tyrosine phosphorylation on insulin receptor $[3,4]$. FFAs activate the inflammatory cascades through several mediators such as toll-like receptors, endoplasmic reticulum stress, and NLRP3 inflammasome, which eventually also converge on JNK and IKK $\beta$ activation [5].

In obesity, activation of inflammatory pathways was later observed in several other tissues, including liver, muscle, hypothalamus, pancreas and gut. Peripheral blood mononuclear cells are also in a proinflammatory state and increase in number [6]. The expression level of proinflammatory genes is increased in obese liver. The number of resident macrophages in the liver (Kupffer cells) does not change in obesity but the activity is increased [7]. Increased MCP-1 expression in obese liver recruits CCR2 ${ }^{+}$myeloid cells which contribute to the development of hepatosteatosis [8]. In obesity, muscle inflammation is induced by increased production of TNFa, IL- $1 \beta$ and IL-6 secreted from accumulated intramuscular adipose tissue. Increased production of proinflammatory cytokines has been observed in obese hypothalamus [9]. Microglia, the resident macrophages in the brain, can be activated by proinflammatory signals and secrete proinflammatory cytokines that act locally on neurons in the hypothalamus to promote leptin resistance and central insulin resistance. The number of macrophages has also been found to increase in obese pancreatic islets which may be attributable to increased IL- 8 production [10]. IL- $1 \beta$ can be secreted by islets and cause $\beta$-cell apoptosis, therefore impairs insulin secretion [11]. Changes of bacteria populations in the intestine and leaky epithelial layer of gut in obesity add an additional source of inflammatory factors such as lipopolysaccharide (LPS) [12].

\section{Initiation of metabolic inflammation and adipose immune cells}

How increase of adiposity signals to immune cells is not completely understood. Multiple types of proinflammatory immune cells have been found to increase in adipose tissue during the development of obesity (Figure 1). Among these cell types, which include macrophages, CD8 ${ }^{+} \mathrm{T}$ lymphocytes, IFN- $\gamma^{+}$Th1 cells (a subtype of CD4 ${ }^{+} \mathrm{T}$ cells), B cells, mast cells and neutrophils, macrophages are the first type of immune cells identified in obese adipose tissue and have been demonstrated to play a critical role in systemic insulin resistance and type 2 diabetes [2,13]. Macrophages can be subdivided into classically activated macrophages (M1) and alternatively activated macrophages (M2). M1 macrophages secrete proinflammatory cytokines whereas M2 macrophages secrete anti-inflammatory cytokines. In vivo, tissue macrophages can be in varied polarization states and span a spectrum between M1 and M2 phenotypes. Adipose tissue macrophages (ATMs) in obese state are M1-like macrophages and are thought to be initially recruited into adipose tissue by chemokines secreted from stressed adipocytes in response to lipid overload. Upon arrival at adipose tissue, these M1 macrophages secrete a large amount of proinflammatory cytokines and chemokines which attract more macrophages, therefore forming a vicious cycle to amplify adipose tissue inflammation. ATMs also promote adipose fibrosis by stimulating the accumulation of collagen and other extracellular matrix components [14]. Animal models with impaired macrophage-mediated inflammatory pathways, such as macrophage specific deficiency of JNK1, IKK $\beta$ and fatty acid binding protein 4, demonstrate improved insulin 
sensitivity in obese state. Ablation of macrophage population also improves insulin sensitivity in diet-induced obese mice $[15,16]$. In contrast, absence of anti-inflammatory peroxisome proliferator-activated receptor $\gamma(\mathrm{PPAR} \gamma)$ in macrophages promotes insulin resistance and glucose intolerance. One function of infiltrated macrophages is perhaps to clear dead adipocytes since M1-like macrophages usually surround dead adipocytes and form a crown-like structure (CLS). Controversial data exist regarding the timing of macrophage infiltration during diet-induced obesity and its role in insulin resistance upon acute high fat diet feeding.

$\mathrm{CD} 8^{+} \mathrm{T}$ lymphocytes and IFN $-\gamma^{+} \mathrm{CD} 4^{+} \mathrm{Th} 1$ cells are believed to promote insulin resistance through recruitment and activation of M1 macrophages in adipose tissue of obese mice $[17,18]$. However, it is controversial whether T cell infiltration precedes macrophage influx [13]. B cells have been shown to modulate activation of $\mathrm{T}$ cells and proinflammatory macrophages as well as produce pathogenic IgG antibodies, therefore inducing insulin resistance in diet-induced obese mice. B cell deficient mice have improved homeostasis depite weight gain [19]. Mast cells are thought to induce insulin resistance through releases of IL- 6 and IFN- $\gamma$. Mast cell deficient mice are resistant to diet-induced obesity, have improved inflammatory profile in adipose tissue and enhanced glucose homeostasis [20]. Stabilization of mast cells by disodium cromoglycate has similar effect [20]. Neutrophils are found to transiently infiltrate abdominal but not subcutaneous adipose tissue and the timing is prior to macrophage influx [21]. Circulating neutrophils in severely obese subjects are found in an proinflammatory state [22]. It remains to be determined by using loss-offunction genetic animal models whether neutrophils play a role in adipose tissue and systemic inflammation and contribute to the development of insulin resistance in obesity.

In addition to increased proinflammatory immune cells, decreased number of antiinflammatory immune cells also contributes to the development of adipose inflammation in obesity (Figure 1). The types of anti-inflammatory immune cells that have been identified in obese adipose tissue include M2 macrophages, eosinophils, regulatory T cells (Treg), invariant natural killer T (iNKT) cells and myeloid-derived suppressor cells (MDSCs). The first four types of cells decrease while MDSCs increase in obesity. M2 macrophages secrete anti-inflammatory cytokines such as IL-10 and IL-1 receptor antagonist [23]. Both M1 and M2 macrophages express F4/80 and CD11b but M1 macrophages are CD11 $\mathrm{c}^{+}$while M2 macrophages are $\mathrm{CD} 11 \mathrm{c}^{-}$. Eosinophils are the major cell type expressing anti-inflammatory IL-4 in adipose tissue and have been shown to improve obesity-related insulin resistance and glucose intolerance by promoting alternative activation of macrophages [24]. Treg cells are potent suppressors of both innate and adaptive immune responses by production of IL-4, IL-10 and IL-13 [25]. Approaches that upregulate Treg cells lead to improvement of insulin sensitivity and glucose tolerance $[18,26]$. Consistently, depletion of Treg cells in lean mice causes insulin resistance and increased levels of proinflammatory cytokines in both adipose tissue and the circulation [26]. iNKT cells are bridging immune cells sparsely represented in adipose tissue [5]. The role of iNKT cells in improving obesity-related insulin resistance is currently in debate due to controversial results from different studies [5]. MDSCs are precursors of mature monocytes and possess the capability to suppress inflammation through attenuating activation of $\mathrm{CD}^{+} \mathrm{T}$ cells, promoting macrophage polarization to the antiinflammatory M2 phenotype, stimulating the development of Treg cells, and abolishing NK cell cytotoxicity [27].

\section{The role of metabolic inflammation in obesity-related diseases}

Obesity is considered as a risk factor for a large array of diseases. Due to space restriction, we will only briefly discuss a few of them including type 2 diabetes (T2D), cardiovascular disease (CVD), fatty liver disease, and cancer. There is a high correlation between obesity 
and T2D. More than eighty percent of T2D patients are obese. Human population studies have demonstrated elevated circulating levels of numerous inflammatory factors in T2D patients. These inflammatory factors range from acute-phase proteins (such as c-reactive protein, haptoglobin, fibrinogen, serum amyloid A and plasminogen activator inhibitor), sialic acid, to proinflammatory cytokines and chemokines [28]. Insulin resistance induced by the inflammatory component is the underlying mechanism for the development of T2D. Abdominal visceral adipose tissue, not subcutaneous adipose tissue, is correlated with insulin resistance. Proinflammatory cytokines such as TNFa, IL-1 $\beta$, and MCP-1 as well as FFAs have been reported to impair insulin signaling. The effect of IL-6 is tissue-specific, inducing insulin resistance in adipose tissue and liver but being beneficial in skeletal muscle [13]. Overall obesity results in impaired insulin responses in insulin target tissues. Insulin resistance causes attenuated insulin-stimulated glucose uptake in skeletal muscle and adipose tissue as well as increased glucose output from the liver. As a response, pancreatic $\beta$ cells secrete more insulin to compensate but eventually fail to keep up with the demand for insulin, therefore leading to the onset of T2D.

The leading causes of mortality in obese and diabetic patients are cardiovascular complications, which impact heart, large vessels (cardiomyopathy and atherosclerosis) and small vessels (neuropathy, retinopathy and nephropathy). Multiple mechanisms, such as lipotoxicity, cardiac and endothelial insulin resistance, inflammation and hyperglycemia, have been proposed. Among these pathways, lipotoxicity has been considered to play an important role in cardiovascular complications [29]. In insulin resistance sate, the ability of insulin in glucose homeostasis is impaired but its lipogenic capability is not harmed. Therefore, hyperinsulinemia promotes ectopic lipid accumulation in cardiomyocytes and endothelial cells. Elevated circulating lipids, increased accumulation of intracellular lipids in cardiac and vascular system, as well as epicardial or pericardial fat depot contribute to lipotoxicity, which eventually lead to cardiac and endothelial dysfunction.

Hepatosteatosis is commonly observed in obese subjects and can often develop into nonalcoholic fatty liver disease (NAFLD) which manifest symptoms ranging from mild hepatosteatosis, steatohepatitis, fibrosis, to cirrhosis, and occasionally, hepatocellular carcinoma [30]. Increased accumulation of lipids in hepatocytes, activation of hepatic inflammatory pathways and infiltration of proinflammatory immune cells play a potential role in obesity-related NAFLD [31]. It remains to be determined whether hepatic inflammation is mediated by resident Kupffer cells or infiltrated immune cells.

Obesity has also been linked to increased risks for developing colorectal, thyroid and cervical cancer [32]. Increased mortality rates in certain cancers are also attributable to obesity (such as prostate and stomach cancer in men; breast, endometrium, cervix, uterus and ovary cancer in women). Elevated circulating levels of IGF-1 and systemic chronic inflammation in obesity are considered as important risk factors for cancer development [32]. In obesity, visceral adipose tissues derived proangiogenic factors, such as VEGF and PAI-1, may also promote neovascularization in tumors. Bariatric surgery induced weight loss is associated with reduced cancer rates [33]. Obesity may predispose can cer patients resistant to therapeutic agents targeting for growth.

\section{Targeting inflammation for improving metabolic profile}

Extensive investigations have established a causal role for metabolic inflammation in obesity-related insulin resistance and type 2 diabetes. The only class of insulin sensitizer on the diabetes market, thiazolidinediones (TZDs), has been shown to act through adipose tissue by activating PPAR $\gamma$, which is expressed in both adipocytes and macrophages in adipose tissue. TZDs enhance adipose tissue lipid storage capacity by promoting 
differentiation of insulin sensitive small adipocytes and repress secretion of inflammatory cytokines. The effects of TZDs can be partially attributable to PPAR $\gamma$ activation in macrophages and Treg cells. Activation of macrophage PPAR $\gamma$ promotes macrophage phenotype switching from proinflammatory M1 to anti-inflammatory M2 and mice with macrophage specific deletion of PPAR $\gamma$ lose the full response to treatment of TZDs [34,35]. PPAR $\gamma$ has also been reported to promote accumulation of anti-inflammatory Treg cells in visceral adipose tissue and PPAR $\gamma$ expression in this cell type is necessary for the complete insulin-sensitizing effect of pioglitazone [36].

Other anti-inflammation reagents, such as salsalate, anakinra, anti-IL-6 receptor antibody, $\mathrm{TNFa}$ antagonizing antibody, and $\mathrm{TNFa}$ receptor-Fc fusion protein have been tested in obese and/or diabetic subjects. Salsalate is a prodrug of salicylate which has been used to treat arthritis for years and considered safe. Salsalate has been shown to reduce glycated albumin and increase circulating adiponectin levels after one month of treatment in a small pilot study involving 20 obese nondiabetic young adults. Later in a three-month study involving 104 obese diabetic subjects, salsalate significantly improved glycemic control and lowered triglyceride levels [6]. Recently, a large National Institutes of Health-funded one year clinical trial of salsalate was completed and the release of the results will provide more insights regarding the effects of salsalate in improving obesity-related type 2 diabetes. Anakinra is an antagonist of human IL-1 receptor. In a 13-week trial involving 70 patients, anakinra was shown to significantly reduce glycated hemoglobin, improve $\beta$-cell secretory function and decrease systemic inflammatory markers [37]. IL-6 antagonism was shown to improve $\mathrm{HbA} 1 \mathrm{c}$ in a 6-month trial involving 39 patients [38]. In contrast, TNFa antagonism failed to improve glycemia in obese diabetic patients and only has a mild effect on insulin sensitivity in insulin-resistant nondiabetic patients [6].

\section{Conclusions and future directions}

Immunometabolism is a recent concept to reflect the intimate relationship between metabolic and inflammatory signaling pathways. In obesity, chronic inflammation has been accepted as a causal factor leading to insulin resistance and other metabolic disorders. Immunotherapies have also been shown to improve obesity-related metabolic disorders. More efficacious therapeutic approaches targeting metabolic inflammation are needed to properly repress pathological inflammatory responses without comprising the normal function of the immune system. To achieve this, in depth studies are needed to investigate the triggering factors of initial inflammation, the interactions and relative importance of different types of immune cells in promoting adipose inflammation, and the downstream signaling events.

\section{Acknowledgments}

Our work is supported by NIH grant R01 DK 080746 (to H.X.) and American Heart Association Scientist Development grant $0830190 \mathrm{~N}$ (to H.X.).

\section{References}

1. Hotamisligil GS, et al. Adipose expression of tumor necrosis factor-alpha: direct role in obesitylinked insulin resistance. Science. 1993; 259 (5091):87-91. [PubMed: 7678183]

2. $\mathrm{Xu} \mathrm{H}$, et al. Chronic inflammation in fat plays a crucial role in the development of obesity-related insulin resistance. J Clin Invest. 2003; 112 (12):1821-1830. [PubMed: 14679177]

3. Gao Z, et al. Serine phosphorylation of insulin receptor substrate 1 by inhibitor kappa B kinase complex. J Biol Chem. 2002; 277 (50):48115-48121. [PubMed: 12351658]

4. Lee YH, et al. c-Jun N-terminal kinase (JNK) mediates feedback inhibition of the insulin signaling cascade. J Biol Chem. 2003; 278 (5):2896-2902. [PubMed: 12417588] 
5. Schipper HS, et al. Adipose tissue-resident immune cells: key players in immunometabolism. Trends Endocrinol Metab. 2012; 23 (8):407-415. [PubMed: 22795937]

6. Romeo GR, et al. Metabolic syndrome, insulin resistance, and roles of inflammation - mechanisms and therapeutic targets. Arterioscler Thromb Vasc Biol. 2012; 32 (8):1771-1776. [PubMed: 22815343]

7. Cai D, et al. Local and systemic insulin resistance resulting from hepatic activation of IKK-beta and NF-kappaB. Nat Med. 2005; 11 (2):183-190. [PubMed: 15685173]

8. Obstfeld AE, et al. C-C chemokine receptor 2 (CCR2) regulates the hepatic recruitment of myeloid cells that promote obesity-induced hepatic steatosis. Diabetes. 2010; 59 (4):916-925. [PubMed: 20103702]

9. De Souza CT, et al. Consumption of a fat-rich diet activates a proinflammatory response and induces insulin resistance in the hypothalamus. Endocrinology. 2005; 146 (10):4192-4199. [PubMed: 16002529]

10. Ehses JA, et al. Increased number of islet-associated macrophages in type 2 diabetes. Diabetes. 2007; 56 (9):2356-2370. [PubMed: 17579207]

11. Maedler K, et al. Glucose-induced beta cell production of IL-1beta contributes to glucotoxicity in human pancreatic islets. J Clin Invest. 2002; 110 (6):851-860. [PubMed: 12235117]

12. Cani PD, et al. Changes in gut microbiota control metabolic endotoxemia-induced inflammation in high-fat diet-induced obesity and diabetes in mice. Diabetes. 2008; 57 (6):1470-1481. [PubMed: 18305141]

13. Osborn O, Olefsky JM. The cellular and signaling networks linking the immune system and metabolism in disease. Nat Med. 2012; 18 (3):363-374. [PubMed: 22395709]

14. Sun K, et al. Adipose tissue remodeling and obesity. J Clin Invest. 2011; 121 (6):2094-2101. [PubMed: 21633177]

15. Feng B, et al. Clodronate liposomes improve metabolic profile and reduce visceral adipose macrophage content in diet-induced obese mice. PLoS One. 2011; 6 (9):e24358. [PubMed: 21931688]

16. Patsouris D, et al. Ablation of CD11c-positive cells normalizes insulin sensitivity in obese insulin resistant animals. Cell Metab. 2008; 8 (4):301-309. [PubMed: 18840360]

17. Nishimura $\mathrm{S}$, et al. CD8+ effector T cells contribute to macrophage recruitment and adipose tissue inflammation in obesity. Nat Med. 2009; 15 (8):914-920. [PubMed: 19633658]

18. Winer $\mathrm{S}$, et al. Normalization of obesity-associated insulin resistance through immunotherapy. Nat Med. 2009; 15 (8):921-929. [PubMed: 19633657]

19. Winer DA, et al. B cells promote insulin resistance through modulation of T cells and production of pathogenic IgG antibodies. Nat Med. 2011; 17 (5):610-617. [PubMed: 21499269]

20. Liu J, et al. Genetic deficiency and pharmacological stabilization of mast cells reduce diet-induced obesity and diabetes in mice. Nat Med. 2009; 15 (8):940-945. [PubMed: 19633655]

21. Elgazar-Carmon V, et al. Neutrophils transiently infiltrate intra-abdominal fat early in the course of high fat feeding. J Lipid Res. 2008

22. Nijhuis J, et al. Neutrophil activation in morbid obesity, chronic activation of acute inflammation. Obesity (Silver Spring). 2009; 17 (11):2014-2018. [PubMed: 19390527]

23. Ouchi N, et al. Adipokines in inflammation and metabolic disease. Nat Rev Immunol. 2011; 11 (2):85-97. [PubMed: 21252989]

24. Wu D, et al. Eosinophils sustain adipose alternatively activated macrophages associated with glucose homeostasis. Science. 2011; 332 (6026):243-247. [PubMed: 21436399]

25. Tiemessen MM, et al. CD4+CD25+Foxp3+ regulatory T cells induce alternative activation of human monocytes/macrophages. Proc Natl Acad Sci U S A. 2007; 104 (49):19446-19451. [PubMed: 18042719]

26. Feuerer M, et al. Lean, but not obese, fat is enriched for a unique population of regulatory $\mathrm{T}$ cells that affect metabolic parameters. Nat Med. 2009; 15 (8):930-939. [PubMed: 19633656]

27. Sun S, et al. Mechanisms of inflammatory responses in obese adipose tissue. Annu Rev Nutr. 2012; 32:261-286. [PubMed: 22404118] 
28. Donath MY, Shoelson SE. Type 2 diabetes as an inflammatory disease. Nat Rev Immunol. 2011; 11 (2):98-107. [PubMed: 21233852]

29. Wende AR, et al. Mechanisms of lipotoxicity in the cardiovascular system. Curr Hypertens Rep. 2012; 14 (6):517-531. [PubMed: 23054891]

30. Garg A, Misra A. Hepatic steatosis, insulin resistance, and adipose tissue disorders. J Clin Endocrinol Metab. 2002; 87 (7):3019-3022. [PubMed: 12107193]

31. Lumeng CN, Saltiel AR. Inflammatory links between obesity and metabolic disease. J Clin Invest. 2011; 121 (6):2111-2117. [PubMed: 21633179]

32. Hursting SD, Hursting MJ. Growth signals, inflammation, and vascular perturbations: mechanistic links between obesity, metabolic syndrome, and cancer. Arterioscler Thromb Vasc Biol. 2012; 32 (8):1766-1770. [PubMed: 22815342]

33. Ashrafian H, et al. Metabolic surgery and cancer: protective effects of bariatric procedures. Cancer. 2011; 117 (9):1788-1799. [PubMed: 21509756]

34. Odegaard JI, et al. Macrophage-specific PPARgamma controls alternative activation and improves insulin resistance. Nature. 2007; 447 (7148):1116-1120. [PubMed: 17515919]

35. Hevener AL, et al. Macrophage PPAR gamma is required for normal skeletal muscle and hepatic insulin sensitivity and full antidiabetic effects of thiazolidinediones. J Clin Invest. 2007; 117 (6): 1658-1669. [PubMed: 17525798]

36. Cipolletta D, et al. PPAR-gamma is a major driver of the accumulation and phenotype of adipose tissue Treg cells. Nature. 2012; 486 (7404):549-553. [PubMed: 22722857]

37. Larsen CM, et al. Interleukin-1-receptor antagonist in type 2 diabetes mellitus. N Engl J Med. 2007; 356 (15):1517-1526. [PubMed: 17429083]

38. Ogata A, et al. Improvement of HbA1c during treatment with humanised anti-interleukin 6 receptor antibody, tocilizumab. Ann Rheum Dis. 2011; 70 (6):1164-1165. [PubMed: 20980285]

Drug Discov Today Dis Mech. Author manuscript; available in PMC 2014 June 01. 


\section{Lean adipose}

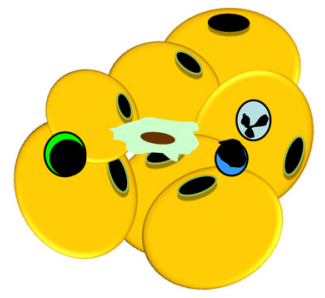

- $\quad$ M2 macrophage

(8) Eosinophil

Treg

inKT cell
Excessive energy intake $+$

Sedentary life style

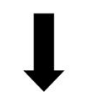

Adipocyte hypertrophy and hyperplasia

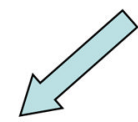

In muscle:

Impairs glucose uptake

Promotes lipid accumulation

Attenuates fatty acid oxidation

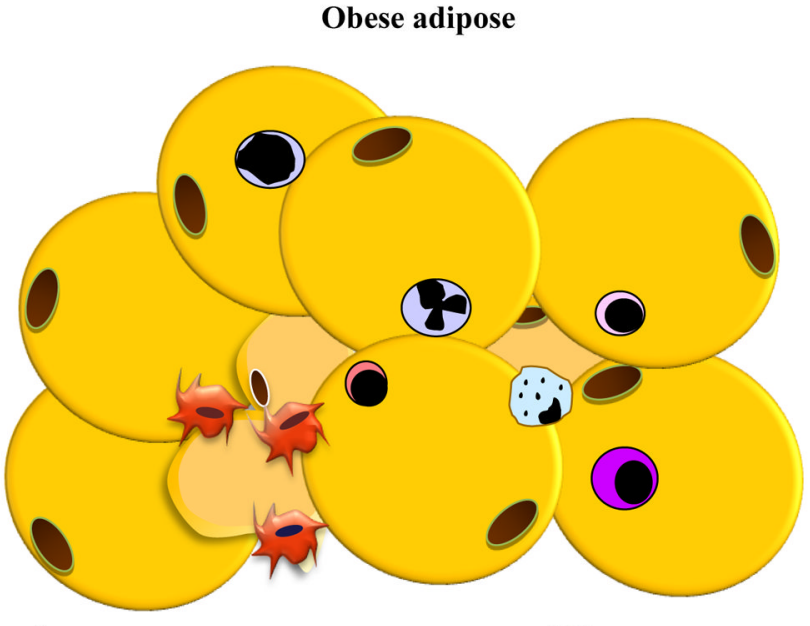

FFA, TNF, IL-1 $\beta$, MCP-1 Neutrophil

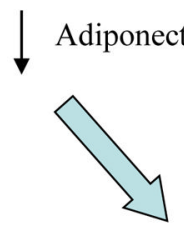

In liver:

Elevates glucose output

Increases lipid accumulation

Diminishes fatty acid oxidation $\because$ Mast cell

B2 cell

CD $8^{+} \mathrm{T}$ cell

IFN- $\gamma^{+}$Th1 cell

Mr M1 macrophage

MDSC

Figure 1.

Illustration of adipose tissue expansion and associated changes of immune cell populations as well as the consequence on systemic insulin resistance. MDSC, myeloid-derived suppressor cell. 